\title{
CPP-GIANT MAGNETORESISTANCE AND THERMOELECTRIC POWER OF MULTILAYERS
}

\author{
S. KROMPIEWSKI* \\ Institute of Molecular Physics, Polish Academy of Sciences \\ Smoluchowskiego 17, 60-179 Poznan, Poland
}

AND U. KREY

Institut für Physik II, Universität Regensburg, 93040 Regensburg, Germany

\begin{abstract}
Oscillations of magnetoresistance and thermoelectric power vs. both nonmagnetic spacer and ferromagnetic slab thicknesses are studied in the current-perpendicular-to-plane geometry, in terms of the single-band tight-binding model. The spin-dependent conductance was calculated from the Kubo formula by means of a recursion Green function technique, and the thermoelectric power directly from the well-known Onsager relations. In general, the observed oscillations may have either just one or two periods. In the latter case the long period of oscillations, related to spectacular beats, is apparently of non-RKKY type. The relative thermoelectric power oscillations are strongly enhanced in comparison with those of the giant magnetoresistance, have the same periods, but different phases and a negative bias.
\end{abstract}

PACS numbers: 75.70.Pa, 75.70.Cn, 75.70.-i

\section{Introduction}

Since the discovery of giant magnetoresistance (GMR) in magnetic multilayers $[1,2]$ there has been a great deal of interest in studying this unusual phenomenon also by theoretical methods [3-7]. The GMR was first interpreted phenomenologically with emphasis put on the role of interface roughness [3], afterwards quantum mechanical methods were applied, too (e.g. [4]). There is no doubt now that the GMR does appear even in systems without any imperfections, since this was clearly demonstrated by ab initio computations in Ref. [5]. In the present paper we study the current-perpendicular-to-plane (CPP)-GMR effect in layered systems of the type $W / F / S / F / W$, where $W$ stands for a semi-infite ideal lead wire, $F$ for a ferromagnet, and $S$ for the non-magnetic spacer.

\footnotetext{
*Corresponding author.
} 


\section{Method and results}

The calculation technique which we developed, is based on the Green function recursion method $[8,6]$. After having performed the Fourier transformation in the $x-y$ plane, the equation of motion of the Green function was reduced (for sc structure) to a simple tridiagonal eigenproblem quite easy to cope with. The conductance of electrons of spin $\sigma$ is given by the Kubo formula, which in turn may be expressed in terms of Green's functions as follows:

$$
\Gamma_{\sigma}=\frac{8 e^{2}}{h} \frac{N_{x} N_{y}}{(2 \pi)^{2}} \int_{\mathrm{BZ}} \mathrm{d}^{2} k_{\|}\left[G_{\sigma}^{\prime \prime}(i, i) G_{\sigma}^{\prime \prime}(i-1, i-1)-G_{\sigma}^{\prime \prime}(i, i-1)^{2}\right] .
$$

Here the index $i$ labels the $z$-planes, $G^{\prime \prime}$ stands for the imaginary part of $G, e^{2} / h$ is the conductance quantum, $N_{x} N_{y}$ - the cross-section area, and the integration is over the 2-dimensional Brillouin zone. Hereafter, both the hopping and the lattice constant are taken as energy- and length units. In addition to the conductance we also calculated the thermoelectric power $S$, which is related to $\Gamma$ by the following well-known formula:

$$
S_{\sigma}=-\pi^{2} k_{\mathrm{B}}^{2}[T /(3|e|)](\mathrm{d} / \mathrm{d} E) \log \Gamma_{\sigma}(E) .
$$

Analogously to the GMR, we define the corresponding "giant magneto-thermoelectric power" by

$$
\text { GMTEP }=\left(S_{\uparrow}^{\uparrow \uparrow}+S_{\downarrow}^{\uparrow \uparrow}\right) /\left(S_{\uparrow}^{\uparrow \downarrow}+S_{\downarrow}^{\uparrow \downarrow}\right)-1,
$$

where the superscripts refer to the parallel and antiparallel magnetization configurations of the two ferromagnets, whereas the subscripts refer to the carrier spin. It can be seen from Fig. 1 that the CPP-GMR oscillates as a function of the thickness $n_{f}$ of the ferromagnetic slab with a short period of $\approx 2$ monolayers (ML). Additionally, in Fig. 1 pronounced beats are seen with typical repetition lengths of roughly $10 \mathrm{ML}$. In case of Fig. 2 with the spacer thickness $\left(n_{s}\right)$ being varied, one gets an intermediate period of $\approx 4.5 \mathrm{ML}$, without any beats. It is clear from these figures that the oscillations of the GMTEP are even stronger than those of the GMR, although they have a negative bias and a different phase than those of the GMR. In any case the GMR oscillations decay always roughly as $n_{f}^{-1}$.

It was shown in [7] that the oscillatory behaviour of $\Gamma$ may arise both from wave numbers with stationary $k_{z}\left(k_{x}, k_{y} ; E_{f}\right)$, as well as from particular cut-off wave numbers $\left(k_{x}, k_{y}\right)$, for which a spectral density vanishes abruptly at one of the ferromagnet/spacer interfaces.

One can roughly estimate the "RKKY-type" wave numbers $k_{z}$ from the asymptotic equation (valid actually for large thicknesses and constant potentials)

$$
k_{z}\left(k_{x}, k_{y} ; E_{f}\right)=\arccos \left[\left(V_{\sigma}-E_{f}\right) / 2-\cos k_{x}-\cos k_{y}\right] .
$$

By finding the extremum with respect to $k_{x}, k_{y}$, with $E_{\mathrm{F}}=2.5$, we get $k_{z}^{(1)}=0.722$ for $V_{\sigma}=0$ (for all the carriers within the spacer and minority-spin electrons in the ferromagnets), and $k_{z}^{(2)}=1.721$ for $V_{\uparrow}=-1.8$ (the majority-spin carriers in the ferromagnets). The corresponding wavelengths can then be calculated as in [9] from the expression $\lambda(p, q)=\pi /\left(p k_{z}^{(1)}+q k_{z}^{(2)}\right)$, which yields $\lambda(0,1)=1.825(V=$ -1.8 ), i.e. roughly the short period of two monolayers (2 ML) seen in Fig. 1. It is 


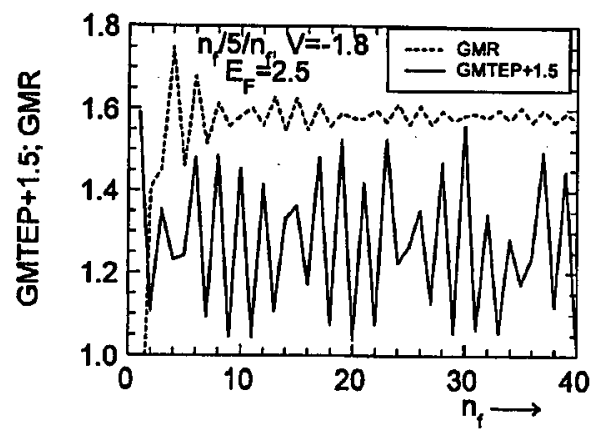

Fig. 1. CPP-GMR (dashed) and GMTEP of the system $n_{f} F / n_{s} S / n_{f} F$ with $n_{s}=5$ (where $F$ and $S$ stand for ferromagnet and spacer, respectively) sandwiched between two semi-infinite ideal lead wires. Majority spin electrons have the potential $V_{\uparrow}=-1.8$ in the ferromagnet (all other potentials are 0 ), $E_{\mathrm{F}}=2.5$.

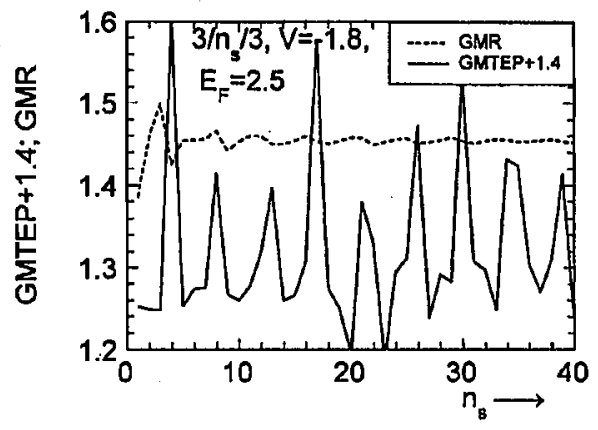

Fig. 2. The same as Fig. 1, but with $n_{f}=3$ on both sides, as a function of the spacer thickness $n_{s}$.

interesting that in Fig. 2, where only the spacer thickness is varied, only a longer wavelength of $\approx 4.5 \mathrm{ML}$ is visible, which would correspond to $\lambda(1,0)=4.35$.

In order to get the beats seen in Fig. 1 one would need, apart from the wave characterized by $k_{z}^{(2)}$, another wave with a wave number very close to it. As there is no way to get such a wave number from Eq. (4), we suppose it to be of non-RKKY character. More insight into the nature of such non-RKKY oscillations can only be gained by taking into account localized and resonance states in addition to extended ones used for the sketchy estimations above. Although the localized states do not contribute directly to the conductance, certainly they modify the density of states and thereby its value at $E_{\mathrm{F}}$. Those unextended states are easily seen in our method as singularities (spread over a large range of energy) of the local densities of states - we are going to study them analytically in the nearest future.

In conclusion, within an $s$-band tight-binding model combined with the Green function rccursion method, we reduced the calculation of the CPP-GMR 
effectively to one dimension. We computed the CPP-GMR for perfect systems composed of two ferromagnets separated by a non-magnetic spacer and sandwiched between ideal infinite lead wires. Unusual beats of the GMR vs. the thickness of the ferromagnetic slab thickness were found and suggested to be due to non-RKKY-type oscillations. Similar results, with even stronger beats, were also obtained for the "giant magneto-thermoelectric power".

\section{Acknowledgments}

This work was carried out under the bilateral project DFG/PAN 436 POL. We also thank the Poznan, Munich and Regensburg Computer Centres for computing time.

\section{References}

[1] M.N. Baibich, J.M. Broto, A. Fert, F.N. Van Dau, F. Petroff, P. Etienne, G. Creuzet, A. Friedel, J. Chazelas, Phys. Rev. Lett. 61, 2472 (1988).

[2] G. Binasch, P. Grünberg, W. Zinn, Phys. Rev. B 39, 4828 (1989).

[3] R.E. Camley, J. Barnaś, Phys. Rev. Lett. 63, 664 (1989).

[4] P.M. Levy, S. Zhang, A. Fert, Phys. Rev. Lett. 65, 1643 (1990).

[5] K.M. Schep, P.J. Kelly, G.E.W. Bauer, Phys. Revं. Lett. 74, 586 (1995).

[6] Y. Asano, A. Oguri, S. Maekawa, Phys. Rev. B 48, 6192 (1993).

[7] J. Mathon, M. Villeret, H. Itoh, Phys. Rev. B 52, R69 (1995).

[8] P.A. Lee, D.S. Fisher, Phys. Rev. Lett. 47, 882 (1981).

[9] J. d'Albuquerque e Castro, J. Mathon, M. Villeret, D.M. Edwards, Phys. Rev. B 51, 12876 (1995). 